\title{
ON THE COMPLETE BOUNDEDNESS OF THE SCHUR BLOCK PRODUCT
}

\author{
ERIK CHRISTENSEN
}

\begin{abstract}
We give a Stinespring representation of the Schur block product on pairs of square matrices with entries in a $\mathrm{C}^{*}$-algebra as a completely bounded bilinear operator of the form:$$
A:=\left(a_{i j}\right), B:=\left(b_{i j}\right): A \square B:=\left(a_{i j} b_{i j}\right)=V^{*} \lambda(A) F \lambda(B) V,
$$

such that $V$ is an isometry, $\lambda$ is a ${ }^{*}$-representation and $F$ is a self-adjoint unitary. This implies an inequality due to Livshits and 2 more ones, apparently new, on the diagonals of matrices:

$$
\begin{aligned}
\|A \square B\| & \leq\|A\|_{r}\|B\|_{c} \text { operator, row and column norm; } \\
-\operatorname{diag}\left(A^{*} A\right) & \leq A^{*} \square A \leq \operatorname{diag}\left(A^{*} A\right), \\
\forall \Xi, \Gamma \in \mathbb{C}^{n} \otimes H:|\langle(A \square B) \Xi, \Gamma\rangle| & \leq\left\|\left(\operatorname{diag}\left(B^{*} B\right)\right)^{1 / 2} \Xi\right\|\left\|\left(\operatorname{diag}\left(A A^{*}\right)\right)^{1 / 2} \Gamma\right\| .
\end{aligned}
$$
\end{abstract}

\section{INTRODUCTION}

The Hadamard or Schur product between a pair of scalar matrices of the same shape has been studied for more than 100 years [16, 8, and it is closely related to basic mathematical subjects such as matrix theory and representation theory. The product also has a natural interest for operator theorists [2, operator algebraists 14] and it is also used in the study of quantum channels, 11. The usage of the names Hadamard and Schur in connection with this product has varied in the literature, and nice expositions on the history behind the use of the names are to be found in Horn [7, section 2 and Horn \& Johnson [8, section 5.0.

In connection with the theory of operator spaces and completely bounded mappings on operator algebras [5, 6, 13, 15] it is obvious to ask questions on the generalization of the Schur product to square matrices over a $\mathrm{C}^{*}$-algebra. This extension of the classical Hadamard or Schur product already exists in the theory for matrices and linear algebra 10, 11, 12, and our present article extends especially results by Horn, Mathias and Nakamura from [11] and Livshits [12. We will return to this point, when we have established some more notation.

In 4] we discussed a bilinear mapping $\Phi$, defined on the product of a pair of $\mathrm{C}^{*}$-algebras $\mathcal{A}, \mathcal{B}$ and mapping into a $\mathrm{C}^{*}$-algebra $\mathcal{C}$. We defined $\Phi$ to be completely bounded if there exists a positive constant $K$ such that for any natural number $k$ and for the bilinear operator $\Phi^{k}$ defined on the $k \times k$ matrices over the algebras

Date: November 12, 2018.

2010 Mathematics Subject Classification. Primary: 15A69, 46L07, 81P68. Secondary: 46N50, 47L25, 81T05.

Key words and phrases. Matrix, Schur product, Hadamard product, operator space, completely bounded, quantum channel. 
$\mathcal{A}, \mathcal{B}$ denoted as $M_{k}(\mathcal{A})$ and $M_{k}(\mathcal{B})$ into $M_{k}(\mathcal{C})$ by (1.1)

$$
\forall A \in M_{k}(\mathcal{A}) \forall B \in M_{k}(\mathcal{B}) \forall i, j \in\{1,2, \ldots, k\}:\left(\Phi^{k}(A, B)\right)_{i j}:=\sum_{l=1}^{k} \Phi\left(a_{i l}, b_{l j}\right),
$$

we have $\left\|\Phi^{k}\right\| \leq K$. If a bilinear operator $\Phi$ is completely bounded we define its completely bounded norm by $\|\Phi\|_{c b}:=\sup \left\{\left\|\Phi^{k}\right\|\right\}$. The main result of the article [4] is that a bilinear operator like $\Phi$ on a pair of $\mathrm{C}^{*}$-algebras $\mathcal{A}, \mathcal{B}$ into a $\mathrm{C}^{*}$-algebra $\mathcal{C}$ acting on a Hilbert space $H$, is completely bounded if and only if there exist Hilbert spaces $K, L,{ }^{*}$-representations $\lambda$ of $\mathcal{A}$ on $K, \rho$ of $\mathcal{B}$ on $L$ and bounded operators $X$ in $B(K, H), Y$ in $B(L, K), Z$ in $B(H, L)$ such that

$$
\forall a \in \mathcal{A} \forall b \in \mathcal{B}: \quad \Phi(a, b)=X \lambda(a) Y \rho(b) Z, \text { and }\|\Phi\|_{c b}=\|X\|\|Y\|\|Z\| .
$$

The decomposition of the bilinear operator $\Phi$ given in (1.2) is called a Stinespring representation of $\Phi$ in recognition of Stinespring's description of completely positive mappings on $\mathrm{C}^{*}$-algebras, 18 .

A linear operator between operator spaces is defined to be completely bounded if all the natural extensions to matrices over the space are bounded by some fixed number. Given a scalar $n \times n$ matrix $A=\left(a_{i j}\right) \in M_{n}(\mathbb{C})$, it is known [6, 17] that the mapping $S_{A}$ on $M_{n}(\mathbb{C})$ which is induced by Schur multiplication with $A$ on $M_{n}(\mathbb{C})$ is completely bounded and the completely bounded norm $\left\|S_{A}\right\|_{c b}$ equals its norm $\left\|S_{A}\right\|$. The aim of this article is to prove that for any operator algebra $\mathcal{A}$ the associative product $\square$ on the algebra $M_{n}(\mathcal{A})$ of $n \times n$ matrices over $\mathcal{A}$, which usually is called the Schur product and is defined by

$$
\forall A, B \in M_{n}(\mathcal{A}): \quad A \square B:=\left(a_{i j} b_{i j}\right),
$$

is completely bounded with completely bounded norm 1 , and that it has a natural decomposition as a difference of 2 positive, a term which will be explained below, bilinear mappings. We do this by providing an explicit and - in our opinion natural - Stinespring representation of $\square$ as a completely bounded bilinear operator on $M_{n}(\mathcal{A})$ of norm 1.

Here we pose a warning to avoid too much confusion. The operation $\square$ is defined on $M_{n}(\mathcal{A})$ and is for any natural number $k$ lifted to an associative product $\square^{k}$ on $M_{k}\left(M_{n}(\mathcal{A})\right)$ via the formula (1.1), so we prove that $\sup \left\{\left\|\square^{k}\right\|\right\}$ is 1 by showing that if the algebra $\mathcal{A}$ acts on a Hilbert space $H$, and $M_{n}(\mathcal{A})$ acts on $\mathbb{C}^{n} \otimes H$, in the natural way, then there exists an isometry $V$ of $\mathbb{C}^{n} \otimes H$ into $\mathbb{C}^{n} \otimes H \otimes \mathbb{C}^{n}$, a selfadjoijnt unitary $F$ on $\mathbb{C}^{n} \otimes H \otimes \mathbb{C}^{n}$ and a unital ${ }^{*}$-representation $\lambda$ of $M_{n}(B(H))$ on $\mathbb{C}^{n} \otimes H \otimes \mathbb{C}^{n}$ such that

$$
\forall A, B \in M_{n}(\mathcal{A}): \quad A \square B=V^{*} \lambda(A) F \lambda(B) V .
$$

Since the self-adjoint unitary is a diifference of 2 complementary orthogonal projections $F=P-(I-P)$, we get from the equation (1.3) that

$$
\forall A, B \in M_{n}(\mathcal{A}): \quad A \square B=V^{*} \lambda(A) P \lambda(B) V-V^{*} \lambda(A)(I-P) \lambda(B) V,
$$

so the Schur block product is written in a natural way as a difference of 2 completely bounded bilinear operators which, in a natural way, may be called positive. It turns out that if we take the absolute value, which we denote $|\square|$, in the sense that we replace $F$ by $I$ in (1.3) then we get 


$$
\forall A, B \in M_{n}(\mathcal{A}): \quad A|\square| B=V^{*} \lambda(A B) V=\operatorname{diag}(A B):=\sum_{1 \leq i, j \leq n} e_{i i} \otimes a_{i j} b_{j i} .
$$

From the equations (1.4) and ( 1.5) we get immediately the following operator inequality

$$
\forall A \in M_{n}(\mathcal{A}): \quad-\operatorname{diag}\left(A^{*} A\right) \leq A^{*} \square A \leq \operatorname{diag}\left(A^{*} A\right),
$$

and an inequality which is closely related to the classical Cauchy-Schwarz inequality for positive semidefinite bilinear forms,

$$
\begin{aligned}
& \forall \Xi, \Gamma \in \mathbb{C}^{n} \otimes H \forall A, B \in M_{n}(B(H)): \\
& \begin{aligned}
|\langle(A \square B) \Xi, \Gamma\rangle|^{2} & \leq\left\langle\operatorname{diag}\left(B^{*} B\right) \Xi, \Xi\right\rangle\left\langle\operatorname{diag}\left(A A^{*}\right) \Gamma, \Gamma\right\rangle \\
& =\left\|\left(\operatorname{diag}\left(B^{*} B\right)\right)^{1 / 2} \Xi\right\|^{2}\left\|\left(\operatorname{diag}\left(A A^{*}\right)\right)^{1 / 2} \Gamma\right\|^{2} .
\end{aligned}
\end{aligned}
$$

After we completed the first draft of a presentation of this result we realized that the operation of constructing $\square^{k}$ was introduced in [11] by Horn, Mathias and Nakamura in the case when the Schur product is the classical one on $M_{n}(\mathbb{C})$, or in other words when the $\mathrm{C}^{*}$-algebra equals $\mathbb{C}$. The article [11] is from 1991, and the quoted result on completely bounded bilinear operators is presented in [4] from 1987. On the other hand the result of Lemma 3.2 of the article [11] actually is related to our description of the Schur product $\square$ given in Theorem 2.9 as a completely bounded operator.

The proof we present below goes back to the classical result for scalar matrices, which tells that the Schur or Hadamard product may be found as a principal submatrix of the Kronecker product, or in modern terms the tensor product, of the two matrices. This result is presented in [8], Lemma 5.1.1. The new twist is that for block matrices the Schur product is no longer commutative.

The reason why we tried to show complete boundedness of the Schur product came from an inequality in [3], where we studied commutators of the form $[f(D), a]$ where $D$ is an unbounded self-adjoint operator and $f$ is an absolutely continuous function with a certain growth condition. The basic tool we used in that study is a result on the operator norm of the Schur product between a pair of operator valued matrices. This result follows easily from the description of the Schur product we give below, and is presented as a part of our main theorem. In the article [3] we got the result as a generalization of Theorem 1.1 point (i) of [2, in which Bennett studies the scalar Schur product. Later on, when working on the present article, we realized that Livshits already in [12 from 1994 published the same inequality. Furthermore the inequality may be seen as an extension of results by Horn, Mathias and Nakamura 9, 11 on analogies to the Cauchy-Schwarz inequality. In order to formulate this result we have to introduce the concepts row norm and column norm of a matrix of operators. The column norm of a matrix with operator entries is simply the supremum of the norms of the columns from the matrix, when considered as operators. The row norm is defined in the obvious analogous way, and it equals the column norm of the adjoint operator. The fundamental norm identity for bounded operators on Hilbert spaces states that $\left\|x^{*} x\right\|=\|x\|^{2}$, and based on this we can give the following formal definition. 
Definition 1.1. Let $\mathcal{A}$ be subalgebra of a $\mathrm{C}^{*}$-algebra $\mathcal{C}, J$ a set of indices and $A=\left(a_{i j}\right), i, j \in J, a_{i j} \in \mathcal{A}$ an $\mathcal{A}$ valued matrix over $J$. The column norm $\|A\|_{c}$ and the row norm $\|A\|_{\mathrm{r}}$ are given by the expressions

$$
\begin{aligned}
& \|A\|_{\mathrm{r}}:=\sup _{i \in J} \sqrt{\left\|\sum_{j \in J} a_{i j} a_{i j}^{*}\right\|}=\sqrt{\left\|\operatorname{diag}\left(A A^{*}\right)\right\|} \\
& \|A\|_{c}:=\sup _{j \in J} \sqrt{\left\|\sum_{i \in J} a_{i j}^{*} a_{i j}\right\|}=\sqrt{\left\|\operatorname{diag}\left(A^{*} A\right)\right\|} .
\end{aligned}
$$

Livshits' inequality may then be presented as follows:

For any pair of matrices $A=\left(a_{i j}\right), B=\left(b_{i j}\right)$ indexed over $J$ and with entries from an operator algebra $\mathcal{A}$, the operator norm $\|A \square B\|$ of the Schur block product $A \square B=\left(a_{i j} b_{i j}\right)$ satisfies the inequality

$$
\|A \square B\| \leq\|A\|_{\mathrm{r}}\|B\|_{\mathrm{c}}
$$

\section{The explicit Stinespring form of the Schur Block Product}

We will now present our decomposition of the Schur block product for matrices over an operator algebra $\mathcal{A}$, and we may and will just as well assume that $\mathcal{A}$ is a subalgebra of $B(H)$ for some Hilbert space $H$. The set of indices $J$, with respect to which we will construct square matrices over $\mathcal{A}$ may be any set and hence it may be infinite. We will use the symbol $M_{J}(\mathcal{A})$ to denote all square matrices over $\mathcal{A}$, indexed by $J$, and defining bounded operators on $\ell^{2}(J, H)$. The point of having $M_{J}(\mathcal{A})$ in mind instead of the larger algebra $M_{J}((B(H))$ is to underline that the Schur block product is an associative product on the algebra $M_{J}(\mathcal{A})$. On the other hand the description of the product we are going to give will be independent of the algebra $\mathcal{A}$, so in the rest of the article we will just consider the Schur block product as a binary operation on matrices over $B(H)$. The result that the Schur product of bounded matrices is bounded, follows directly from the description of the product we give.

We will first define the notation we are using. There is a canonical orthonormal basis say $\left\{\alpha_{j}: j \in J\right\}$ for $\ell^{2}(J, \mathbb{C})$ and corresponding to this basis there is a set of matrix units $e_{i j}$ in $B\left(\ell^{2}(J, \mathbb{C})\right)$ such that we have $e_{i j} \alpha_{k}=\delta_{j k} \alpha_{i}$. We will then adopt the notation that for a matrix $A=\left(a_{i j}\right)$ in $M_{J}(B(H))$ we will represent it as an operator on the Hilbert space $\ell^{2}(J, \mathbb{C}) \otimes H \otimes \ell^{2}(J, \mathbb{C})$ in 3 different ways as limits of strongly convergent bounded nets, such that each element in each of the nets is a finite sum of elementary tensor products of operators. The ordered index set for all 3 nets will be denoted $(\mathcal{F}(J), \subseteq)$, and it consists of all finite subsets of $J$ ordered by inclusion.

$$
\begin{aligned}
& \lambda(A):=\underset{F \in \mathcal{F}(J)}{\operatorname{strong~limit}} \sum_{i, j, k \in F} e_{i j} \otimes a_{i j} \otimes e_{k k}, \\
& \sigma(A):=\underset{F \in \mathcal{F}(J)}{\operatorname{strong~limit}} \sum_{i, j \in F} e_{i j} \otimes a_{i j} \otimes e_{i j}, \\
& \rho(A):=\underset{F \in \mathcal{F}(J)}{\operatorname{strong~limit}} \sum_{i, k, l \in F} e_{i i} \otimes a_{k l} \otimes e_{k l} .
\end{aligned}
$$

We assume that it is well known that the mappings $\lambda, \rho, \sigma$ are faithful *-representations, i.e. injective self-adjoint homomorphisms of norm 1 . In particular this 
means that for 2 bounded matrices $A=\left(a_{i j}\right)$ and $B=\left(b_{i j}\right)$ and for a fixed pair $(i, m)$ of indices the infinite sum $\sum_{j \in J} a_{i j} b_{j m}$ is strongly convergent and defines a matrix element $c_{i m}$ of a matrix $C$ in $M_{J}(B(H))$, such that $\lambda(C)$ is given as the strong limit

$$
\begin{aligned}
\lambda(C) & =\underset{F \in \mathcal{F}(J)}{\operatorname{strong} \operatorname{limit}} \sum_{i, j, k, l, m, n \in F}\left(e_{i j} \otimes a_{i j} \otimes e_{k k}\right)\left(e_{l m} \otimes b_{l m} \otimes e_{n n}\right) \\
& =\underset{F \in \mathcal{F}(J)}{\operatorname{strong} \operatorname{limit}} \sum_{i, j, k, m \in F} e_{i m} \otimes a_{i j} b_{j m} \otimes e_{k k} .
\end{aligned}
$$

The representations $\lambda$ and $\rho$ are unital, but $\sigma$ is not, unless $J$ consists of one element. For $\sigma$ we get $\sigma(I)=\sum_{j \in J} e_{j j} \otimes I_{B(H)} \otimes e_{j j}$ which is an orthogonal projection, say $Q$, from $\left.\ell^{2}(J, \mathbb{C}) \otimes H \otimes \ell^{2}(J, \mathbb{C})\right)$ onto the closed subspace $K$ which is spanned by all the vectors of the form $\left\{\alpha_{j} \otimes \xi \otimes \alpha_{j}: j \in J, \xi \in H\right\}$. The reason why we have attached the names $\lambda, \rho$ and $\sigma$ to these representations, is that in the case when $H=\mathbb{C}$ and $J=\{1, \ldots, n\}$, then $\lambda$ and $\rho$ are named the left and the right standard representation of $M_{n}(\mathbb{C})$ in the theory of von Neumann algebras and these 2 representations have something in common with the left and the right regular representation of a discrete group. The representation $\sigma$ is a kind of symmetric mix and it fits nicely into the description of the Schur block product. Before we can see that, we need a generalization of the Kronecker product to the setting of matrices with operator entries.

Definition 2.1. Let $A=\left(a_{i j}\right)$ and $B=\left(b_{k l}\right)$ be elements in $M_{J}((B(H))$. The Kronecker block product of $A$ and $B$ is the matrix $A *_{K B} B$ in $M_{J}\left(M_{J}(B(H))\right)$, which is defined by the equation

$$
A *_{K B} B:=\lambda(A) \rho(B)=\underset{F \in \mathcal{F}(J)}{\operatorname{strong~limit}} \sum_{i, j, k, l \in F} e_{i j} \otimes a_{i j} b_{k l} \otimes e_{k l} .
$$

We may now benefit from the classical result 8 Lemma 5.1.1, which describes the Schur product of two scalar matrices as a principal submatrix of their Kronecker product. In the setting of (2.6), the matrix, we are looking at, is of the form $(J \times J) \times(J \times J)$ and the rows are indexed by pairs $(i, k)$ whereas the columns are indexed by pairs $(j, l)$ and the principal submatrix, which gives the Schur block product, is the one where the index set consists of all the pairs $\{(j, j): j \in J\}$. Moreover we find right away that the orthogonal projection $Q$ we defined above is exactly the one which supports the principal sub-matrices which have non-zero entries only on elements which have indices of the form $((i, i),(j, j))$. Based on this we state without any further proof the following proposition:

Proposition 2.2. Let $A=\left(a_{i j}\right)$ and $B=\left(b_{i j}\right)$ be elements in $M_{J}((B(H))$, then their Schur block product $A \square B=\left(a_{i j} b_{i j}\right)$ is in $M_{J}((B(H))$ and

$$
\sigma(A \square B)=\underset{F \in \mathcal{F}(J)}{\text { strong limit }} \sum_{i j \in F} e_{i j} \otimes a_{i j} b_{i j} \otimes e_{i j}=Q \lambda(A) \rho(B) Q=Q\left(A *_{K B} B\right) Q .
$$

It should be remarked, that you may right away see, that in the case when $H=\mathbb{C}$, then the matrices $A=\left(a_{i j}\right)$ and $B=\left(b_{k l}\right)$ are scalar matrices, and the Kronecker block product is just the well known Kronecker product. 
The space $K:=Q\left(\ell^{2}(J, \mathbb{C}) \otimes H \otimes \ell^{2}(J, \mathbb{C})\right)$ is closely related to $\ell^{2}(J, \mathbb{C}) \otimes H$ and we define an isometry $V$ of $\ell^{2}(J, \mathbb{C}) \otimes H$ onto $K$ by

$$
\forall \Xi \in \ell^{2}(J, \mathbb{C}) \otimes H, \Xi=\sum_{j \in J} \alpha_{j} \otimes \xi_{j}: \quad V \Xi:=\sum_{j \in J} \alpha_{j} \otimes \xi_{j} \otimes \alpha_{j} .
$$

It is now a matter of computation to verify the following equation, which shows that the representation $\sigma$ on $K$ is unitarily equivalent to the identity representation of $M_{J}(B(H))$ on $\ell^{2}(J, \mathbb{C}) \otimes H$.

$$
\forall A=\left(a_{i j}\right) \in M_{J}(B(H)): \quad \sigma(A) V=V A, \text { or } A=V^{*} \sigma(A) V .
$$

We may then present our first theorem.

Theorem 2.3. The Schur block product is completely bounded, with completely bounded norm 1.

Proof. We give a description of the Schur block product in the form described in equation (1.2), so let $A=\left(a_{i j}\right)$ and $B=\left(b_{i j}\right)$ be in $M_{J}(B(H))$ then

$$
\begin{aligned}
A \square B & =V^{*} \sigma(A \square B) V \text { by (2.9) } \\
& =V^{*}\left(A *_{K B} B\right) V \text { by (2.7) and } V V^{*}=Q \\
& =V^{*} \lambda(A) \rho(B) V \text { by (2.6), }
\end{aligned}
$$

and we have obtained the form from (1.2) with operators of norm 1.

Since $M_{J}(B(H))$ has a unit, the completely bounded norm is 1 , and the theorem follows.

We could leave the result like this, but we think that the bilinear operators, we look at, do have many things in common with sesquilinear forms, and in the latter case we do prefer self-adjoint or even better positive semidefinite forms. A similar kind of aesthetics may apply here, so we want to describe the Schur block product not only as a bilinear completely bounded operator, but rather as a difference of 2 positive completely bounded bilinear operators. We are not far from this in the equation (2.10), but we need to introduce a well known self-adjoint unitary to get the expression, we think may be the right one.

Definition 2.4. The flip operator $F$ on $\ell^{2}(J, \mathbb{C}) \otimes H \otimes \ell^{2}(J, \mathbb{C})$ is defined as the strong limit

$$
F:=\underset{K \in \mathcal{F}(J)}{\operatorname{strong} \operatorname{limit}} \sum_{i, j \in F} e_{i j} \otimes I \otimes e_{j i} .
$$

We have a couple of simple observations, which we collect in the following lemma.

\section{Lemma 2.5.}

(i) $F$ is a self-adjoint unitary

(ii) $\forall A=\left(a_{i j}\right) \in M_{J}(B(H)): \quad F \lambda(A) F=\rho(A)$

(iii) $F V=V$.

Proof. It is well known that $F$ is a self-adjoint unitary, which has the property that for $X, Z$ in $B\left(\ell^{2}(J, \mathbb{C})\right)$ and $Y$ in $B(H)$ we have $F(X \otimes Y \otimes Z) F=Z \otimes Y \otimes X$, so the statements (i) and (ii) follow. The statement (iii) follows easily once we remark that the subspace $K=Q\left(\ell^{2}(J, \mathbb{C}) \otimes H \otimes \ell^{2}(J, \mathbb{C})\right)$, which is the range space of $V$, is spanned by vectors of the form $\alpha_{i} \otimes \xi \otimes \alpha_{i}$ and all such vectors are clearly eigenvectors for $F$ corresponding to the eigenvalue 1. 
Below we list a property of the isometry $V$, which will show why the operator norm of a Schur product $A \square B$ is related to the row norm of $A$ and the column norm of $B$ as described in (1.8).

Lemma 2.6. For any matrix $A=\left(a_{i j}\right)$ in $M_{J}(B(H))$ we have

$$
\begin{aligned}
& \|A\|_{c}=\|\lambda(A) V\| \\
& \|A\|_{\mathrm{r}}=\left\|V^{*} \lambda(A)\right\| .
\end{aligned}
$$

Proof. We will prove the column case only, since the row case follows by taking adjoints. First remark, that $Q=V V^{*}$, so the identity $\|\lambda(A) Q\|=\|\lambda(A) V\|$ follows because $V$ is an isometry. Then let us compute the square of the norm $\lambda(A) Q$ of using the $\mathrm{C}^{*}$-algebraic norm identity.

$$
\begin{aligned}
& \|\lambda(A) Q\|^{2}=\left\|Q \lambda\left(A^{*} A\right) Q\right\| \\
& =\| \underset{F \in \mathcal{F}(J)}{\| \operatorname{strong} \operatorname{limit}}\left(\sum_{i, j, k, l, s, t \in F}\left(e_{i i} \otimes I \otimes e_{i i}\right)\left(e_{j l} \otimes a_{k j}^{*} a_{k l} \otimes e_{s s}\right)\left(e_{t t} \otimes I \otimes e_{t t}\right) \|\right.
\end{aligned}
$$

we see that $i=j=s=t=l$ so

$$
\begin{aligned}
& \leq \limsup _{F \in \mathcal{F}(J)}\left\|\sum_{i, k \in F} e_{i i} \otimes a_{k i}^{*} a_{k i} \otimes e_{i i}\right\| \\
& =\sup _{i \in J}\left\|\sum_{k \in J} a_{k i}^{*} a_{k i}\right\|=\|A\|_{c}^{2} .
\end{aligned}
$$

On the other hand, for each $j \in J$ we have

$$
\|\lambda(A) Q\| \geq\left\|\lambda(A)\left(e_{j j} \otimes I_{B(H)} \otimes e_{j j}\right)\right\|=\sqrt{\left\|\sum_{i \in J} a_{i j}^{*} a_{i j}\right\|},
$$

so $\|\lambda(A) Q\| \geq\|A\|_{c}$ and the lemma follows.

We give the formal definition of the diagonal of a matrix of operators

Definition 2.7. For an operator $A=\left(a_{i j}\right)$ in $M_{J}(B(H))$ we will define the diagonal $\operatorname{diag}(A)$ in $M_{J}(B(H))$ by

$$
\operatorname{diag}(A)_{i j}=\left\{\begin{array}{ll}
0 & \text { if } i \neq j \\
a_{i i} & \text { if } i=j
\end{array} .\right.
$$

There are some simple observations we will use.

Lemma 2.8. For an operator $A$ in $M_{J}(B(H))$ we have

$$
\begin{aligned}
\operatorname{diag}(A) & =V^{*} \lambda(A) V \\
\|A\|_{c} & =\left\|\operatorname{diag}\left(A^{*} A\right)\right\|^{1 / 2} \\
\|A\|_{r} & =\left\|\operatorname{diag}\left(A A^{*}\right)\right\|^{1 / 2} .
\end{aligned}
$$

Then we can state the main result.

Theorem 2.9. For any Hilbert space $H$ and any set of indices $J$ :

(i) The Schur block product on $M_{J}(B(H))$ is given by the formula.

$$
\forall A, B \in M_{J}(B(H)): \quad A \square B=V^{*} \lambda(A) F \lambda(B) V .
$$


(ii) This is Livshits's inequality, 12 .

$$
\forall A, B \in M_{J}(B(H)): \quad\|A \square B\| \leq\|A\|_{r}\|B\|_{c} .
$$

(iii) Let $X=\left(x_{i j}\right)$, and $Y=\left(y_{i j}\right)$ be matrices indexed by $J$ with elements from $B(H)$, and $C$ a non negative real. If the matrix $Z$ defined by $Z=$ $\left(z_{i j}\right):=\left(x_{i j} y_{i j}\right)$ is bounded whenever $Y$ is column bounded and satisfies $\|Z\|_{o p} \leq C\|Y\|_{c}$, then $X$ is row bounded and satisfies $\|X\|_{r} \leq C$.

$$
\forall A \in M_{J}(B(H)):-\operatorname{diag}\left(A^{*} A\right) \leq A^{*} \square A \leq \operatorname{diag}\left(A^{*} A\right) .
$$

(v)

$$
\begin{aligned}
& \forall \Xi, \Gamma \in \ell^{2}(J, \mathbb{C}) \otimes H \forall A, B \in M_{J}(B(H)): \\
& |\langle(A \square B) \Xi, \Gamma\rangle| \leq\left\|\left(\operatorname{diag}\left(B^{*} B\right)\right)^{1 / 2} \Xi\right\|\left\|\left(\operatorname{diag}\left(A A^{*}\right)\right)^{1 / 2} \Gamma\right\| .
\end{aligned}
$$

Proof. For $A, B$ in $M_{J}(B(H))$ we have

$$
\begin{aligned}
A \square B & =V^{*} \lambda(A) \rho(B) V \text { by (2.10) } \\
& =V^{*} \lambda(A) F \lambda(B) F V \text { by Lemma 2.5 (ii) } \\
& =V^{*} \lambda(A) F \lambda(B) V \text { by Lemma 2.5 (iii), }
\end{aligned}
$$

and the claim (i) is proved.

The claim (ii) follows from (i) and the result in Lemma 2.6.

Item (iii) shows that Livshits's inequality determines the row norm and by symmetry the column norm as well. To prove it, let $k$ be in $J$, then we can estimate the norm of the $k^{\prime}$ th row of $X$ via the assumptions made. We define the matrix $Y$ by $y_{i j}=0$ if $i \neq k, j \in J$ and $y_{k j}=1$ for all $j$ in $J$. Then $Y$ is column bounded with column norm 1 and the norm of the matrix $Z:=\left(x_{i j} y_{i j}\right)$ is exactly the norm of the $k$ 'th row of $X$.

The statement in (iv) is a direct consequence of (i), Lemma 2.8 and the fact that $-\lambda\left(A^{*} A\right) \leq \lambda\left(A^{*}\right) F \lambda(A) \leq \lambda\left(A^{*} A\right)$.

With respect to item (v), we find from the classical Cauchy-Schwarz inequality, the statement in (i) and the Lemma 2.8 that

$$
\begin{aligned}
\forall \Xi, \Gamma \in \ell^{2}(J, \mathbb{C}) \otimes H \forall A, B & \in M_{J}(B(H)): \\
|\langle(A \square B) \Xi, \Gamma\rangle|^{2} & =\left|\left\langle V^{*} \lambda(A) F \lambda(B) V \Xi, \Gamma\right\rangle\right|^{2} \\
& \leq\|\lambda(B) V \Xi\|^{2}\left\|\lambda\left(A^{*}\right) V \Gamma\right\|^{2} \\
& =\left\langle V^{*} \lambda\left(B^{*} B\right) V \Xi, \Xi\right\rangle\left\langle V^{*} \lambda\left(A A^{*}\right) V \Gamma, \Gamma\right\rangle \\
& =\left\langle\operatorname{diag}\left(B^{*} B\right) \Xi, \Xi\right\rangle\left\langle\operatorname{diag}\left(A A^{*}\right) \Gamma, \Gamma\right\rangle,
\end{aligned}
$$

and by taking square roots

$$
|\langle(A \square B) \Xi, \Gamma\rangle| \leq\left\|\left(\operatorname{diag}\left(B^{*} B\right)\right)^{1 / 2} \Xi\right\|\left\|\left(\operatorname{diag}\left(A A^{*}\right)\right)^{1 / 2} \Gamma\right\| .
$$




\section{An ELEMENTARY OBSERVATION}

It is worth to remark, that the statement (iv) in Theorem 2.9 above implies Livshits' inequality, and that (iv) is an easy consequence of the ordinary CauchySchwarz inequality as the few lines of computations below show. Hence the validity of the inequality (iv) may have been realized by many people before, but may be not linked to the complete boundedness of the Schur product. In fact we find by 2 applications of Cauchy-Schwarz inequalities for Hilbert spaces and for numbers respectively that for any index set $J$, vectors $\Xi=\left(\xi_{j}\right), \Gamma=\left(\gamma_{j}\right)$, in $\ell^{2}(J, H)$ and bounded matrices of operators $A=\left(a_{i j}\right)$ and $B=\left(b_{i j}\right)$ in $M_{J}(B(H))$ we have

$$
\begin{aligned}
|\langle(A \square B) \Xi, \Gamma\rangle|^{2} & =\left|\sum_{i, j \in J}\left\langle b_{i j} \xi_{j}, a_{i j}^{*} \gamma_{i}\right\rangle\right|^{2} \\
& \leq\left(\sum_{i, j \in J}\left\|b_{i j} \xi_{j}\right\|^{2}\right)\left(\sum_{i, j \in J}\left\|a_{i j}^{*} \gamma_{i}\right\|^{2}\right) \\
& =\left(\sum_{j \in J}\left\langle\sum_{i \in J} b_{i j}^{*} b_{i j} \xi_{j}, \xi_{j}\right\rangle\right)\left(\sum_{i \in J}\left\langle\sum_{j \in J} a_{i j} a_{i j}^{*} \gamma_{i}, \gamma_{i}\right\rangle\right) \\
& =\left\langle\operatorname{diag}\left(B^{*} B\right) \Xi, \Xi\right\rangle\left\langle\operatorname{diag}\left(A A^{*}\right) \Gamma, \Gamma\right\rangle .
\end{aligned}
$$

\section{REFERENCES}

[1] G. Aubrun, S. J. Szarek, Alice and Bob Meet Banach, Amer. Math. Soc. Math. Surv. Monographs, 223 (2017).

[2] G. Bennett, Schur multipliers, Duke Math. J. 44 (1977) , 603 - 639.

[3] E. Christensen, Commutator Inequalities via Schur Products, Abel Symp. 2015, 127 - 144, Springer 2016.

[4] E. Christensen, A. M. Sinclair, Representations of Completely Bounded Multilinear Operators, J. Funct. Anal. 72 (1987), $151-181$.

[5] E. G. Effros, Z.-J: Ruan, Operator Spaces, Oxford UP, 2000.

[6] U. Haagerup, Decomposition of completely bounded maps on operator algebras, Unpublished preprint.

[7] R. A. Horn, The Hadamard product, Proc. Sympos. Appl. Math. 40 (1990). 87 - 169.

[8] R. A. Horn, C. R. Johnson, Topics in matrix analysis, Cambridge UP, 1991

[9] R. A. Horn, R. Mathias, An analog of the Cauchy-Schwarz inequality for Hadamard products and unitarily invariant norms SIAM J. Matrix Anal. Appl. 11 (1990). 481- 496.

[10] R. A. Horn, R. Mathias, Block-matrix generalizations of Schur's basic theorems on Hadamard products, Linear Algebra Appl. 172 (1992). 337- 346.

[11] R. A. Horn., R. Mathias, Y. Nakamura, Inequalities for unitarily invariant norms and bilinear matrix products, Linear Multilinear Algebra 30 (1991), 303- 314.

[12] L. Livshits, Block-matrix generalizations of infinite-dimensional schur products and schur multipliers, Linear Multilinear Algebra 38 (1994), 59-78,

[13] V. Paulsen, Completely bounded maps and operator algebras, Cambridge studies in Math 78, (2002).

[14] G. Pisier, Similarity problems and completely bounded maps, Lecture Notes in Math. 1618 2nd. edition, Springer 2001.

[15] G. Pisier, Introduction to operator space theory, Cambridge UP, 2003.

[16] I. Schur, Bemerkungen zur Theorie der beschränkten Bilinearformen mit unendlich vielen Veränderlichen. J. Reine Angew. Math., 140 1911, 1-28.

[17] R. R. Smith, Completely bounded module maps and the Haagerup tensor product, J. Funct. Anal. 102 (1991), 156 - 175.

[18] W. Stinespring, Positive functions on $C^{*}$-algebras, Proc. Amer. Math. Soc. 6 (1966), 211-216. 
Institute for Mathematical Sciences, Universitetsparken 5, 2100 Copenhagen, DenMARK

E-mail address: echris@math.ku.dk 